Pacific

Journal of

Mathematics

\title{
ACTION OF LONGEST ELEMENT ON A HECKE ALGEBRA
} CELL MODULE

GEORGE LUSZTIG

Volume 279 No. 1-2

December 2015 


\title{
ACTION OF LONGEST ELEMENT ON A HECKE ALGEBRA CELL MODULE
}

\author{
GEORGE LUSZTIG \\ Dedicated to the memory of Robert Steinberg
}

\begin{abstract}
By a result of Mathas, the basis element $T_{w_{0}}$ of the Hecke algebra of a finite Coxeter group acts in the canonical basis of a cell module as a permutation matrix times plus or minus a power of $v$. We generalize this result to the unequal parameter case. We also show that the image of $T_{w_{0}}$ in the corresponding asymptotic Hecke algebra is given by a simple formula.
\end{abstract}

\section{Introduction}

0.1. The Hecke algebra $\mathcal{H}$ (over $\mathcal{A}=\mathbb{Z}\left[v, v^{-1}\right], v$ an indeterminate) of a finite Coxeter group $W$ has two bases as an $\mathcal{A}$-module: the standard basis $\left\{T_{x} ; x \in W\right\}$ and the basis $\left\{C_{x} ; x \in W\right\}$ introduced in [Kazhdan and Lusztig 1979]. The second basis determines a decomposition of $W$ into two-sided cells and a partial order for the set of two-sided cells, see [Kazhdan and Lusztig 1979]. Let $l \rightarrow \mathbb{N}$ be the length function, let $w_{0}$ be the longest element of $W$ and let $c$ be a two-sided cell. Let $a$ (resp. $a^{\prime}$ ) be the value of the $\boldsymbol{a}$-function [Lusztig 2003, 13.4] on $\boldsymbol{c}$ (resp. on $w_{0} \boldsymbol{c}$ ). The following result was proved by Mathas [1996].

(a) There exists a unique permutation $u \mapsto u^{*}$ of $\boldsymbol{c}$ such that for any $u \in \boldsymbol{c}$ we have $T_{w_{0}}(-1)^{l(u)} C_{u}=(-1)^{l\left(w_{0}\right)+a^{\prime}} v^{-a+a^{\prime}}(-1)^{l\left(u^{*}\right)} C_{u^{*}}$ plus an $\mathcal{A}$-linear combination of elements $C_{u^{\prime}}$ with $u^{\prime}$ in a two-sided cell strictly smaller than $\boldsymbol{c}$. Moreover, for any $u \in \boldsymbol{c}$ we have $\left(u^{*}\right)^{*}=u$.

A related (but weaker) result appears in [Lusztig 1984, (5.12.2)]. A result similar to (a) which concerns canonical bases in representations of quantum groups appears in [Lusztig 1990, Corollary 5.9]; now, in the case where $W$ is of type $A$, (a) can be deduced from [loc. cit.] using the fact that irreducible representations of the Hecke algebra of type $A$ (with their canonical bases) can be realized as 0 -weight spaces of certain irreducible representations of a quantum group with their canonical bases.

Supported in part by National Science Foundation grant DMS-1303060.

MSC2010: 20F55, 20G15.

Keywords: Hecke algebra, left cell, Weyl group. 
As R. Bezrukavnikov pointed out to the author, (a) specialized for $v=1$ (in the group algebra of $W$ instead of $\mathcal{H}$ ) and assuming that $W$ is crystallographic can be deduced from [Bezrukavnikov et al. 2012, Proposition 4.1] (a statement about Harish-Chandra modules), although it is not explicitly stated there.

In this paper we shall prove a generalization of (a) which applies to the Hecke algebra associated to $W$ and any weight function assumed to satisfy the properties P1-P15 in [Lusztig 2003, §14], see Theorem 2.3; (a) corresponds to the special case where the weight function is equal to the length function. As an application we show that the image of $T_{w_{0}}$ in the asymptotic Hecke algebra is given by a simple formula (see Corollary 2.8).

0.2. Notation. $W$ is a finite Coxeter group; the set of simple reflections is denoted by $S$. We shall adopt many notations of [Lusztig 2003]. Let $\leq$ be the standard partial order on $W$. Let $l \rightarrow \mathbb{N}$ be the length function of $W$ and let $L \rightarrow \mathbb{N}$ be a weight function (see [Lusztig 2003, 3.1]), that is, a function such that $L\left(w w^{\prime}\right)=$ $L(w)+L\left(w^{\prime}\right)$ for any $w, w^{\prime}$ in $W$ such that $l\left(w w^{\prime}\right)=l(w)+l\left(w^{\prime}\right)$; we assume that $L(s)>0$ for any $s \in S$. Let $w_{0}, \mathcal{A}$ be as in Section 0.1 and let $\mathcal{H}$ be the Hecke algebra over $\mathcal{A}$ associated to $W, L$ as in [Lusztig 2003, 3.2]; we shall assume that properties P1-P15 in [Lusztig 2003, §14] are satisfied. (This holds automatically if $L=l$ by [Lusztig 2003, §15] using the results of [Elias and Williamson 2014]. This also holds in the quasisplit case, see [Lusztig 2003, §16].) We have $\mathcal{A} \subset \mathcal{A}^{\prime} \subset K$ where $\mathcal{A}^{\prime}=\mathbb{C}\left[v, v^{-1}\right], K=\mathbb{C}(v)$. Let $\mathcal{H}_{K}=K \otimes_{\mathcal{A}} \mathcal{H}$ (a $K$ algebra). Recall that $\mathcal{H}$ has an $\mathcal{A}$-basis $\left\{T_{x} ; x \in W\right\}$, see [Lusztig 2003, 3.2] and an $\mathcal{A}$-basis $\left\{c_{x} ; x \in W\right\}$, see [Lusztig 2003, 5.2]. For $x \in W$ we have $c_{x}=\sum_{y \in W} p_{y, x} T_{y}$ and $T_{x}=\sum_{y \in W}(-1)^{l(x y)} p_{w_{0} x, w_{0} y} c_{y}$ (see [Lusztig 2003, 11.4]) where $p_{x, x}=1$ and $p_{y, x} \in v^{-1} \mathbb{Z}\left[v^{-1}\right]$ for $y \neq x$. We define preorders $\leq_{\mathcal{L}}, \leq_{\mathcal{R}}, \leq_{\mathcal{L} R}$ on $W$ in terms of $\left\{c_{x} ; x \in W\right\}$ as in [Lusztig 2003, 8.1]. Let $\sim_{\mathcal{L}}, \sim_{\mathcal{R}}, \sim_{\mathcal{L} \mathcal{R}}$ be the corresponding equivalence relations on $W$, see [Lusztig 2003, 8.1] (the equivalence classes are called left cells, right cells, two-sided cells). Let ${ }^{-}: \mathcal{A} \rightarrow \mathcal{A}$ be the ring involution such that $\overline{v^{n}}=v^{-n}$ for $n \in \mathbb{Z}$. Let ${ }^{-}: \mathcal{H} \rightarrow \mathcal{H}$ be the ring involution such that $\overline{f T_{x}}=\bar{f} T_{x^{-1}}^{-1}$ for $x \in W, f \in \mathcal{A}$. For $x \in W$ we have $\overline{c_{x}}=c_{x}$. Let $h \mapsto h^{\dagger}$ be the algebra automorphism of $\mathcal{H}$ or of $\mathcal{H}_{K}$ given by $T_{x} \mapsto(-1)^{l(x)} T_{x^{-1}}^{-1}$ for all $x \in W$, see [Lusztig 2003, 3.5]. Then the basis $\left\{c_{x}^{\dagger} ; x \in W\right\}$ of $\mathcal{H}$ is defined. (In the case where $L=l$, for any $x$ we have $c_{x}^{\dagger}=(-1)^{l(x)} C_{x}$ where $C_{x}$ is as in Section 0.1.) Let $h \mapsto h^{\text {b }}$ be the algebra antiautomorphism of $\mathcal{H}$ given by $T_{x} \mapsto T_{x^{-1}}$ for all $x \in W$, see [Lusztig 2003, 3.5]; for $x \in W$ we have $c_{x}^{b}=c_{x^{-1}}$, see [Lusztig 2003, 5.8]. For $x, y \in W$ we have $c_{x} c_{y}=\sum_{z \in W} h_{x, y, z} c_{z}, c_{x}^{\dagger} c_{y}^{\dagger}=\sum_{z \in W} h_{x, y, z} c_{z}^{\dagger}$, where $h_{x, y, z} \in \mathcal{A}$. For any $z \in W$ there is a unique number $\boldsymbol{a}(z) \in \mathbb{N}$ such that for any $x, y$ in $W$ we have

$$
h_{x, y, z}=\gamma_{x, y, z^{-1}} v^{\boldsymbol{a}(z)}+\text { strictly smaller powers of } v \text {, }
$$


where $g_{x, y, z^{-1}} \in \mathbb{Z}$ and $g_{x, y, z^{-1}} \neq 0$ for some $x, y$ in $W$. We have also

$$
h_{x, y, z}=\gamma_{x, y, z^{-1}} v^{-a(z)}+\text { strictly larger powers of } v \text {. }
$$

Moreover $z \mapsto \boldsymbol{a}(z)$ is constant on any two-sided cell. The free abelian group $J$ with basis $\left\{t_{w} ; w \in W\right\}$ has an associative ring structure given by $t_{x} t_{y}=\sum_{z \in W} \gamma_{x, y, z^{-1}} t_{z}$; it has a unit element of the form $\sum_{d \in \mathcal{D}} n_{d} t_{d}$ where $\mathcal{D}$ is a subset of $W$ consisting of certain elements with square 1 and $n_{d}= \pm 1$. Moreover for $d \in \mathcal{D}$ we have $n_{d}=\gamma_{d, d, d}$.

For any $x \in W$ there is a unique element $d_{x} \in \mathcal{D}$ such that $x \sim_{\mathcal{L}} d_{x}$. For a commutative ring $R$ with 1 we set $J_{R}=R \otimes J$ (an $R$-algebra).

There is a unique $\mathcal{A}$-algebra homomorphism $\phi: \mathcal{H} \rightarrow J_{\mathcal{A}}$ such that $\phi\left(c_{x}^{\dagger}\right)=$ $\sum_{d \in \mathcal{D}, z \in W ; d_{z}=d} h_{x, d, z} n_{d} t_{z}$ for any $x \in W$. After applying $\mathbb{C} \otimes_{\mathcal{A}}$ to $\phi$ (we regard $\mathbb{C}$ as an $\mathcal{A}$-algebra via $v \mapsto 1), \phi$ becomes a $\mathbb{C}$-algebra isomorphism $\phi_{\mathbb{C}}: \mathbb{C}[W] \stackrel{\sim}{\longrightarrow} J_{\mathbb{C}}$ (see [Lusztig 2003, 20.1(e)]). After applying $K \otimes_{\mathcal{A}}$ to $\phi, \phi$ becomes a $K$-algebra isomorphism $\phi_{K}: \mathcal{H}_{K} \stackrel{\sim}{\longrightarrow} J_{K}$ (see [Lusztig 2003, 20.1(d)]).

For any two-sided cell $\boldsymbol{c}$ let $\mathcal{H}^{\leq c}$ (resp. $\mathcal{H}^{<\boldsymbol{c}}$ ) be the $\mathcal{A}$-submodule of $\mathcal{H}$ spanned by $\left\{c_{x}^{\dagger}, x \in W, x \leq_{\mathcal{L R}} x^{\prime}\right.$ for some $\left.x^{\prime} \in \boldsymbol{c}\right\}$ (resp. $\left\{c_{x}^{\dagger}, x \in W, x<_{\mathcal{L R}} x^{\prime}\right.$ for some $\left.\left.x^{\prime} \in \boldsymbol{c}\right\}\right)$. Note that $\mathcal{H}^{\leq c}, \mathcal{H}^{<c}$ are two-sided ideals in $\mathcal{H}$. Hence $\mathcal{H}^{c}:=\mathcal{H}^{\leq c} / \mathcal{H}^{<c}$ is an $(\mathcal{H}, \mathcal{H})$ bimodule. It has an $\mathcal{A}$-basis $\left\{c_{x}^{\dagger}, x \in \boldsymbol{c}\right\}$. Let $J^{\boldsymbol{c}}$ be the subgroup of $J$ spanned by $\left\{t_{x} ; x \in \boldsymbol{c}\right\}$. This is a two-sided ideal of $J$. Similarly, $J_{\mathbb{C}}^{c}:=\mathbb{C} \otimes J^{c}$ is a two-sided ideal of $J_{\mathbb{C}}$ and $J_{K}^{c}:=K \otimes J^{c}$ is a two-sided ideal of $J_{K}$.

We write $E \in \operatorname{Irr} W$ whenever $E$ is a simple $\mathbb{C}[W]$-module. We can view $E$ as a (simple) $J_{\mathbb{C}}$-module $E_{\boldsymbol{a}}$ via the isomorphism $\phi_{\mathbb{C}}^{-1}$. Then the (simple) $J_{K}$-module $K \otimes_{\mathbb{C}} E_{\bullet}$ can be viewed as a (simple) $\mathcal{H}_{K}$-module $E_{v}$ via the isomorphism $\phi_{K}$. Let $E^{\dagger}$ be the simple $\mathbb{C}[W]$-module which coincides with $E$ as a $\mathbb{C}$-vector space but with the $w$ action on $E^{\dagger}$ (for $w \in W$ ) being $(-1)^{l(w)}$ times the $w$-action on $E$. Let $\boldsymbol{a}_{E} \in \mathbb{N}$ be as in [Lusztig 2003, 20.6(a)].

\section{Preliminaries}

1.1. Let $\sigma: W \rightarrow W$ be the automorphism given by $w \mapsto w_{0} w w_{0}$; it satisfies $\sigma(S)=S$ and it extends to a $\mathbb{C}$-algebra isomorphism $\sigma: \mathbb{C}[W] \rightarrow \mathbb{C}[W]$. For $s \in S$ we have $l\left(w_{0}\right)=l\left(w_{0} s\right)+l(s)=l(\sigma(s))+l\left(\sigma(s) w_{0}\right)$ hence $L\left(w_{0}\right)=L\left(w_{0} s\right)+L(s)=$ $L(\sigma(s))+L\left(\sigma(s) w_{0}\right)=L(\sigma(s))+L\left(w_{0} s\right)$ so that $L(\sigma(s))=L(s)$. It follows that $L(\sigma(w))=L(w)$ for all $w \in W$ and that we have an $\mathcal{A}$-algebra automorphism $\sigma: \mathcal{H} \rightarrow \mathcal{H}$ where $\sigma\left(T_{w}\right)=T_{\sigma(w)}$ for any $w \in W$. This extends to a $K$-algebra isomorphism $\sigma: \mathcal{H}_{K} \rightarrow \mathcal{H}_{K}$. We have $\sigma\left(c_{w}\right)=c_{\sigma(w)}$ for any $w \in W$. For any $h \in \mathcal{H}$ we have $\sigma\left(h^{\dagger}\right)=(\sigma(h))^{\dagger}$. Hence we have $\sigma\left(c_{w}^{\dagger}\right)=c_{\sigma(w)}^{\dagger}$ for any $w \in W$. We have $h_{\sigma(x), \sigma(y), \sigma(z)}=h_{x, y, z}$ for all $x, y, z \in W$. It follows that $\boldsymbol{a}(\sigma(w))=\boldsymbol{a}(w)$ for all $w \in W$ and $\gamma_{\sigma(x), \sigma(y), \sigma(z)}=\gamma_{x, y, z}$ for all $x, y, z \in W$ so that we have a ring 
isomorphism $\sigma: J \rightarrow J$ where $\sigma\left(t_{w}\right)=t_{\sigma(w)}$ for any $w \in W$. This extends to an $\mathcal{A}$-algebra isomorphism $\sigma: J_{\mathcal{A}} \rightarrow J_{\mathcal{A}}$, to a $\mathbb{C}$-algebra isomorphism $\sigma: J_{\mathbb{C}} \rightarrow J_{\mathbb{C}}$ and to a $K$-algebra isomorphism $\sigma: J_{K} \rightarrow J_{K}$. From the definitions we see that $\phi: \mathcal{H} \rightarrow J_{\mathcal{A}}$ (see Section 0.2) satisfies $\phi \sigma=\sigma \phi$. Hence $\phi_{\mathbb{C}}$ satisfies $\phi_{\mathbb{C}} \sigma=\sigma \phi_{\mathbb{C}}$ and $\phi_{K}$ satisfies $\phi_{K} \sigma=\sigma \phi_{K}$. We show:

$$
\text { For } h \in \mathcal{H} \text { we have } \sigma(h)=T_{w_{0}} h T_{w_{0}}^{-1} \text {. }
$$

It is enough to show this for $h$ running through a set of algebra generators of $\mathcal{H}$. Thus we can assume that $h=T_{s}^{-1}$ with $s \in S$. We must show that $T_{\sigma(s)}^{-1} T_{w_{0}}=T_{w_{0}} T_{s}^{-1}$ : both sides are equal to $T_{\sigma(s) w_{0}}=T_{w_{0} s}$.

Lemma 1.2. For any $x \in W$ we have $\sigma(x) \sim_{\mathcal{L} \mathcal{R}} x$.

From 1.1(a) we deduce that $T_{w_{0}} c_{x} T_{w_{0}}^{-1}=c_{\sigma(x)}$. In particular, $\sigma(x) \leq_{\mathcal{L} \mathcal{R}} x$. Replacing $x$ by $\sigma(x)$ we obtain $x \leq_{\mathcal{L R}} \sigma(x)$. The lemma follows.

1.3. Let $E \in \operatorname{Irr} W$. We define $\sigma_{E}: E \rightarrow E$ by $\sigma_{E}(e)=w_{0} e$ for $e \in E$. We have $\sigma_{E}^{2}=1$. For $e \in E, w \in W$, we have $\sigma_{E}(w e)=\sigma(w) \sigma_{E}(e)$. We can view $\sigma_{E}$ as a vector space isomorphism $E_{\bullet} \stackrel{\sim}{\longrightarrow} E_{\boldsymbol{\bullet}}$. For $e \in E_{\bullet}, w \in W$ we have $\sigma_{E}\left(t_{w} e\right)=t_{\sigma(w)} \sigma_{E}(e)$. Now $\sigma_{E}: E_{\star} \rightarrow E_{\star}$ defines by extension of scalars a vector space isomorphism $E_{v} \rightarrow E_{v}$ denoted again by $\sigma_{E}$. It satisfies $\sigma_{E}^{2}=1$. For $e \in E_{v}, w \in W$ we have $\sigma_{E}\left(T_{w} e\right)=T_{\sigma(w)} \sigma_{E}(e)$.

Lemma 1.4. Let $E \in \operatorname{Irr} W$. There is a unique (up to multiplication by a scalar in $K-\{0\})$ vector space isomorphism $g: E_{v} \rightarrow E_{v}$ such that $g\left(T_{w} e\right)=T_{\sigma(w)} g(e)$ for all $w \in W, e \in E_{v}$. We can take for example $g=T_{w_{0}}: E_{v} \rightarrow E_{v}$ or $g=\sigma_{E}: E_{v} \rightarrow E_{v}$. Hence $T_{w_{0}}=\lambda_{E} \sigma_{E}: E_{v} \rightarrow E_{v}$ where $\lambda_{E} \in K-\{0\}$.

The existence of $g$ is clear from the second sentence of the lemma. If $g^{\prime}$ is another isomorphism $g^{\prime}: E_{v} \rightarrow E_{v}$ such that $g^{\prime}\left(T_{w} e\right)=T_{\sigma(w)} g^{\prime}(e)$ for all $w \in W, e \in E_{v}$, then for any $e \in E_{v}$ we have $g^{-1} g^{\prime}\left(T_{w} e\right)=g^{-1} T_{\sigma(w)} g^{\prime}(e)=T_{w} g^{-1} g^{\prime}(e)$ and using Schur's lemma we see that $g^{-1} g^{\prime}$ is a scalar. This proves the first sentence of the lemma hence the third sentence of the lemma.

1.5. Let $E \in \operatorname{Irr} W$. We have

$$
\sum_{x \in W} \operatorname{tr}\left(T_{x}, E_{v}\right) \operatorname{tr}\left(T_{x^{-1}}, E_{v}\right)=f_{E_{v}} \operatorname{dim}(E)
$$

where $f_{E_{v}} \in \mathcal{A}^{\prime}$ is of the form

$$
f_{E_{v}}=f_{0} v^{-2 a_{E}}+\text { strictly higher powers of } v
$$

and $f_{0} \in \mathbb{C}-\{0\}$. (See [Lusztig 2003, 19.1(e), 20.1(c), 20.7].) 
From Lemma 1.4 we see that $\lambda_{E}^{-1} T_{w_{0}}$ acts on $E_{v}$ as $\sigma_{E}$. Using [Lusztig 2005, 34.14(e)] with $c=\lambda_{E}^{-1} T_{w_{0}}$ (an invertible element of $\mathcal{H}_{K}$ ) we see that

$$
\sum_{x \in W} \operatorname{tr}\left(T_{x} \sigma_{E}, E_{v}\right) \operatorname{tr}\left(\sigma_{E}^{-1} T_{x^{-1}}, E_{v}\right)=f_{E_{v}} \operatorname{dim}(E) .
$$

Lemma 1.6. Let $E \in \operatorname{Irr} W$. We have $\lambda_{E}=v^{n_{E}}$ for some $n_{E} \in \mathbb{Z}$.

For any $x \in W$ we have

$$
\operatorname{tr}\left(\sigma_{E} c_{x}^{\dagger}, E_{v}\right)=\sum_{d \in \mathcal{D}, z \in W ; d=d_{z}} h_{x, d, z} n_{d} \operatorname{tr}\left(\sigma_{E} t_{z}, E_{\bullet}\right) \in \mathcal{A}^{\prime}
$$

since $\operatorname{tr}\left(\sigma_{E} t_{z}, E_{\bullet}\right) \in \mathbb{C}$. It follows that $\operatorname{tr}\left(\sigma_{E} h, E_{v}\right) \in \mathcal{A}^{\prime}$ for any $h \in \mathcal{H}$. In particular, both $\operatorname{tr}\left(\sigma_{E} T_{w_{0}}, E_{v}\right)$ and $\operatorname{tr}\left(T_{w_{0}}^{-1} \sigma_{E}, E_{v}\right)$ belong to $\mathcal{A}^{\prime}$. Thus $\lambda_{E} \operatorname{dim} E$ and $\lambda_{E}^{-1} \operatorname{dim} E$ belong to $\mathcal{A}^{\prime}$ so that $\lambda_{E}=b v^{n}$ for some $b \in \mathbb{C}-\{0\}$ and $n \in \mathbb{Z}$. From the definitions we have $\left.\lambda_{E}\right|_{v=1}=1$ (for $v=1, T_{w_{0}}$ becomes $w_{0}$ ) hence $b=1$. The lemma is proved.

Lemma 1.7. Let $E \in \operatorname{Irr} W$. There exists $\epsilon_{E} \in\{1,-1\}$ such that for any $x \in W$ we have

$$
\operatorname{tr}\left(\sigma_{E^{\dagger}} T_{x},\left(E^{\dagger}\right)_{v}\right)=\epsilon_{E}(-1)^{l(x)} \operatorname{tr}\left(\sigma_{E} T_{x^{-1}}^{-1}, E_{v}\right)
$$

Let $\left(E_{v}\right)^{\dagger}$ be the $\mathcal{H}_{K}$-module with underlying vector space $E_{v}$ such that the action of $h \in \mathcal{H}_{K}$ on $\left(E_{v}\right)^{\dagger}$ is the same as the action of $h^{\dagger}$ on $E_{v}$. From the proof in [Lusztig 2003, 20.9] we see that there exists an isomorphism of $\mathcal{H}_{K}$-modules $b:\left(E_{v}\right)^{\dagger} \stackrel{\sim}{\longrightarrow}\left(E^{\dagger}\right)_{v}$. Let $\iota:\left(E_{v}\right)^{\dagger} \rightarrow\left(E_{v}\right)^{\dagger}$ be the vector space isomorphism which corresponds under $b$ to $\sigma_{E^{\dagger}}:\left(E^{\dagger}\right)_{v} \rightarrow\left(E^{\dagger}\right)_{v}$. Then we have $\operatorname{tr}\left(\sigma_{E^{\dagger}} T_{x},\left(E^{\dagger}\right)_{v}\right)=$ $\operatorname{tr}\left(\iota T_{x},\left(E_{v}\right)^{\dagger}\right)$. It is enough to prove that $\iota= \pm \sigma_{E}$ as a $K$-linear map of the vector space $E_{v}=\left(E_{v}\right)^{\dagger}$ into itself. From the definition we have $\iota\left(T_{w} e\right)=T_{\sigma(w)} \iota(e)$ for all $w \in W, e \in\left(E_{v}\right)^{\dagger}$. Hence $(-1)^{l(w)} \iota\left(T_{w^{-1}}^{-1} e\right)=(-1)^{l(w)} T_{\sigma\left(w^{-1}\right)}^{-1}(e)$ for all $w \in W, e \in E_{v}$. It follows that $\iota(h e)=(-1)^{l(w)} T_{\sigma(h)} \iota(e)$ for all $h \in \mathcal{H}, e \in E_{v}$. Hence $\iota\left(T_{w} e\right)=T_{\sigma(w)} \iota(e)$ for all $w \in W, e \in E_{v}$. By the uniqueness in Lemma 1.4 we see that $\iota=\epsilon_{E} \sigma_{E}: E_{v} \rightarrow E_{v}$ where $\epsilon_{E} \in K-\{0\}$. Since $\iota^{2}=1, \sigma_{E}^{2}=1$, we see that $\epsilon_{E}= \pm 1$. The lemma is proved.

Lemma 1.8. Let $E \in \operatorname{Irr} W$. We have $n_{E}=-\boldsymbol{a}_{E}+\boldsymbol{a}_{E^{\dagger}}$.

For $x \in W$ we have (using Lemmas 1.4 and 1.6)

$$
\operatorname{tr}\left(T_{w_{0} x}, E_{v}\right)=\operatorname{tr}\left(T_{w_{0}} T_{x^{-1}}^{-1}, E_{v}\right)=v^{n_{E}} \operatorname{tr}\left(\sigma_{E} T_{x^{-1}}^{-1}, E_{v}\right) .
$$


Making a change of variable $x \mapsto w_{0} x$ in 1.5 (a) and using that $T_{x^{-1} w_{0}}=T_{w_{0} \sigma(x)^{-1}}$ we obtain

$$
\begin{aligned}
f_{E_{v}} \operatorname{dim}(E) & =\sum_{x \in W} \operatorname{tr}\left(T_{w_{0} x}, E_{v}\right) \operatorname{tr}\left(T_{w_{0} \sigma(x)^{-1}}, E_{v}\right) \\
& =v^{2 n_{E}} \sum_{x \in W} \operatorname{tr}\left(\sigma_{E} T_{x^{-1}}^{-1}, E_{v}\right) \operatorname{tr}\left(\sigma_{E} T_{\sigma(x)}^{-1}, E_{v}\right) .
\end{aligned}
$$

Using now Lemma 1.7 and the equality $l(x)=l\left(\sigma\left(x^{-1}\right)\right)$ we obtain

$$
\begin{aligned}
f_{E_{v}} \operatorname{dim}(E) & =v^{2 n_{E}} \sum_{x \in W} \operatorname{tr}\left(\sigma_{E^{\dagger}} T_{x},\left(E^{\dagger}\right)_{v}\right) \operatorname{tr}\left(\sigma_{E^{\dagger}} T_{\sigma\left(x^{-1}\right)},\left(E^{\dagger}\right)_{v}\right) \\
& =v^{2 n_{E}} \sum_{x \in W} \operatorname{tr}\left(\sigma_{E^{\dagger}} T_{x},\left(E^{\dagger}\right)_{v}\right) \operatorname{tr}\left(T_{\xi^{-1}} \sigma_{E^{\dagger}},\left(E^{\dagger}\right)_{v}\right) \\
& =v^{2 n_{E}} f_{\left(E^{\dagger}\right)_{v}} \operatorname{dim}\left(E^{\dagger}\right) .
\end{aligned}
$$

(The last step uses $1.5(\mathrm{c})$ for $E^{\dagger}$ instead of $E$.) Thus we have $f_{E_{v}}=v^{2 n_{E}} f_{\left(E^{\dagger}\right)_{v}}$. The left-hand side is as in 1.5(b) and similarly the right-hand side of the form

$$
f_{0}^{\prime} v^{2 n_{E}-2 a_{E^{\dagger}}}+\text { strictly higher powers of } v
$$

where $f_{0}, f_{0}^{\prime} \in \mathbb{C}-\{0\}$. It follows that $-2 \boldsymbol{a}_{E}=2 n_{E}-2 \boldsymbol{a}_{E^{\dagger}}$. The lemma is proved.

Lemma 1.9. Let $E \in \operatorname{Irr} W$ and let $x \in W$. We have

$$
\begin{aligned}
\operatorname{tr}\left(T_{x}, E_{v}\right) & =(-1)^{l(x)} v^{-a_{E}} \operatorname{tr}\left(t_{x}, E_{\bullet}\right) \quad \bmod v^{-a_{E}+1} \mathbb{C}[v], \\
\operatorname{tr}\left(\sigma_{E} T_{x}, E_{v}\right) & =(-1)^{l(x)} v^{-a_{E}} \operatorname{tr}\left(\sigma_{E} t_{x}, E_{\bullet}\right) \quad \bmod v^{-a_{E}+1} \mathbb{C}[v] .
\end{aligned}
$$

For a proof of (a), see [Lusztig 2003, 20.6(b)]. We now give a proof of (b) along the same lines as that of (a). There is a unique two sided cell $\boldsymbol{c}$ such that $\left.t_{z}\right|_{E_{\bullet}}=0$ for $z \in W-\boldsymbol{c}$. Let $a=\boldsymbol{a}(z)$ for all $z \in \boldsymbol{c}$. By [Lusztig 2003, 20.6(c)] we have $a=\boldsymbol{a}_{E}$. From the definition of $c_{x}$ we see that $T_{x}=\sum_{y \in W} f_{y} c_{y}$, where $f_{x}=1$ and $f_{y} \in v^{-1} \mathbb{Z}\left[v^{-1}\right]$ for $y \neq x$. Applying $\dagger$ we obtain $(-1)^{l(x)} T_{x^{-1}}^{-1}=\sum_{y \in W} f_{y} c_{y}^{\dagger}$; applying ${ }^{-}$we obtain $(-1)^{l(x)} T_{x}=\sum_{y \in W} \bar{f}_{y} c_{y}^{\dagger}$. Thus we have

$$
(-1)^{l(x)} \operatorname{tr}\left(\sigma_{E} T_{x}, E_{v}\right)=\sum_{y \in W} \bar{f}_{y} \operatorname{tr}\left(\sigma_{E} c_{y}^{\dagger}, E_{v}\right)=\sum_{\substack{y, z \in W \\ d \in \mathcal{D} ; d=d_{z}}} \bar{f}_{y} h_{y, d, z} n_{d} \operatorname{tr}\left(\sigma_{E} t_{z}, E_{\bullet}\right) .
$$

In the last sum we can assume that $z \in c$ and $d \in \boldsymbol{c}$ so that $h_{y, d, z}=\gamma_{y, d, z^{-1}} v^{-a}$ $\bmod v^{-a+1} \mathbb{Z}[v]$. Since $\bar{f}_{x}=1$ and $\bar{f}_{y} \in v \mathbb{Z}[v]$ for all $y \neq x$ we see that

$$
(-1)^{l(x)} \operatorname{tr}\left(\sigma_{E} T_{x}, E_{v}\right)=\sum_{\substack{z \in \boldsymbol{c} \\ d \in \mathcal{D} \cap c}} \gamma_{x, d, z^{-1}} n_{d} v^{-a} \operatorname{tr}\left(\sigma_{E} t_{z}, E_{\bullet}\right) \quad \bmod v^{-a+1} \mathbb{C}[v] .
$$


If $x \notin \boldsymbol{c}$ then $\gamma_{x, d, z^{-1}}=0$ for all $d, z$ in the sum so that $\operatorname{tr}\left(\sigma_{E} T_{x}, E_{v}\right)=0$; we have also $\operatorname{tr}\left(\sigma_{E} t_{x}, E_{\boldsymbol{a}}\right)=0$ and the desired formula follows. We now assume that $x \in \boldsymbol{c}$. Then for $d, z$ as above we have $\gamma_{x, d, z^{-1}}=0$ unless $x=z$ and $d=d_{x}$ in which case $\gamma_{x, d, z^{-1}} n_{d}=1$. Thus (b) holds again. The lemma is proved.

Lemma 1.10. Let $E \in \operatorname{Irr} W$. Let $\boldsymbol{c}$ be the unique two sided cell such that $\left.t_{z}\right|_{E_{\boldsymbol{\bullet}}}=0$ for $z \in W-\boldsymbol{c}$. Let $\boldsymbol{c}^{\prime}$ be the unique two sided cell such that $\left.t_{z}\right|_{\left(E^{\dagger}\right)_{n}}=0$ for $z \in W-\boldsymbol{c}^{\prime}$. We have $\boldsymbol{c}^{\prime}=w_{0} \boldsymbol{c}$.

Using 1.8(a) and 1.7(a) we have

$$
\operatorname{tr}\left(T_{w_{0} x}, E_{v}\right)=v^{n_{E}} \operatorname{tr}\left(\sigma_{E} T_{x^{-1}}^{-1}, E_{v}\right)=v^{n_{E}} \epsilon_{E}(-1)^{l(x)} \operatorname{tr}\left(\sigma_{E^{\dagger}} T_{x},\left(E^{\dagger}\right)_{v}\right) .
$$

Using 1.9(a) for $E$ and 1.9(b) for $E^{\dagger}$ we obtain

$$
\begin{array}{rlr}
\operatorname{tr}\left(T_{w_{0} x}, E_{v}\right) & =(-1)^{l\left(w_{0} x\right)} v^{-\boldsymbol{a}_{E}} \operatorname{tr}\left(t_{w_{0} x}, E_{\bullet}\right) & \bmod v^{-\boldsymbol{a}_{E}+1} \mathbb{C}[v], \\
\operatorname{tr}\left(\sigma_{E^{\dagger}} T_{x},\left(E^{\dagger}\right)_{v}\right) & =(-1)^{l(x)} v^{-\boldsymbol{a}_{E^{\dagger}} \operatorname{tr}\left(\sigma_{E^{\dagger}} t_{x}, E_{\bullet}^{\dagger}\right)} & \bmod v^{-\boldsymbol{a}_{E^{\dagger}}+1} \mathbb{C}[v] .
\end{array}
$$

Combining with (a) we obtain

$$
\begin{aligned}
& (-1)^{l\left(w_{0} x\right)} v^{-a_{E}} \operatorname{tr}\left(t_{w_{0} x}, E_{\bullet}\right)+\text { strictly higher powers of } v \\
= & v^{n_{E}} \epsilon_{E} v^{-a_{E^{\dagger}}} \operatorname{tr}\left(\sigma_{E^{\dagger}} t_{x}, E_{\bullet}^{\dagger}\right)+\text { strictly higher powers of } v .
\end{aligned}
$$

Using the equality $n_{E}=-\boldsymbol{a}_{E}+\boldsymbol{a}_{E^{\dagger}}$ (see Lemma 1.8) we deduce

$$
(-1)^{l\left(w_{0} x\right)} \operatorname{tr}\left(t_{w_{0} x}, E_{\bullet}\right)=\epsilon_{E} \operatorname{tr}\left(\sigma_{E^{\dagger}} t_{x}, E_{\bullet}^{\dagger}\right) .
$$

Now we can find $x \in W$ such that $\operatorname{tr}\left(t_{w_{0} x}, E_{\bullet}\right) \neq 0$ and the previous equality shows that $\left.t_{x}\right|_{\left(E^{\dagger}\right)} \neq 0$. Moreover from the definition we have $w_{0} x \in \boldsymbol{c}$ and $x \in \boldsymbol{c}^{\prime}$ so that $w_{0} \boldsymbol{c} \cap \boldsymbol{c}^{\prime} \neq \varnothing$. Since $w_{0} \boldsymbol{c}$ is a two-sided cell (see [Lusztig 2003, 11.7(d)]) it follows that $w_{0} \boldsymbol{c}=\boldsymbol{c}^{\prime}$. The lemma is proved.

Lemma 1.11. Let $\boldsymbol{c}$ be a two-sided cell of $W$. Let $\boldsymbol{c}^{\prime}$ be the two-sided cell $w_{0} \boldsymbol{c}=\boldsymbol{c} w_{0}$ (see Lemma 1.2). Let $a=\boldsymbol{a}(x)$ for any $x \in \boldsymbol{c}$; let $a^{\prime}=\boldsymbol{a}\left(x^{\prime}\right)$ for any $x^{\prime} \in \boldsymbol{c}^{\prime}$. The $K$-linear map $J_{K}^{c} \rightarrow J_{K}^{c}$ given by $\xi \mapsto \phi\left(v^{a-a^{\prime}} T_{w_{0}}\right) \xi$ (left multiplication in $\left.J_{K}\right)$ is obtained from a $\mathbb{C}$-linear map $J_{\mathbb{C}}^{c} \rightarrow J_{\mathbb{C}}^{c}$ (with square 1 ) by extension of scalars from $\mathbb{C}$ to $K$.

We can find a direct sum decomposition $J_{\mathbb{C}}^{c}=\oplus_{i=1}^{m} E^{i}$ where $E^{i}$ are simple left ideals of $J_{\mathbb{C}}$ contained in $J_{\mathbb{C}}^{c}$. We have $J_{K}^{c}=\oplus_{i=1}^{m} K \otimes E^{i}$. It is enough to show that for any $i$, the $K$-linear map $K \otimes E^{i} \rightarrow K \otimes E^{i}$ given by the action of $\phi\left(v^{a-a^{\prime}} T_{w_{0}}\right)$ in the left $J_{K}$-module structure of $K \otimes E^{i}$ is obtained from a $\mathbb{C}$-linear map $E^{i} \rightarrow E^{i}$ (with square 1) by extension of scalars from $\mathbb{C}$ to $K$. We can find $E \in \operatorname{Irr} W$ such that $E^{i}$ is isomorphic to $E_{\boldsymbol{a}}$ as a $J_{\mathbb{C}}$-module. It is then enough to show that the action of $v^{a-a^{\prime}} T_{w_{0}}$ in the left $\mathcal{H}_{K}$-module structure of $E_{v}$ is obtained from the map $\sigma_{E}: E \rightarrow E$ by extension of scalars from $\mathbb{C}$ to $K$. This follows from the equality 
$v^{a-a^{\prime}} T_{w_{0}}=\sigma_{E}: E_{v} \rightarrow E_{v}$ (since $\sigma_{E}$ is obtained by extension of scalars from a $\mathbb{C}$-linear map $E \rightarrow E$ with square 1) provided that we show that $-n_{E}=a-a^{\prime}$. Since $n_{E}=-\boldsymbol{a}_{E}+\boldsymbol{a}_{E^{\dagger}}$ (see Lemma 1.8) it is enough to show that $a=\boldsymbol{a}_{E}$ and $a^{\prime}=a_{E^{\dagger}}$. The equality $a=a_{E}$ follows from [Lusztig 2003, 20.6(c)]. The equality $a^{\prime}=\boldsymbol{a}_{E^{\dagger}}$ also follows from [Lusztig 2003, 20.6(c)] applied to $E^{\dagger}, \boldsymbol{c}^{\prime}=w_{0} \boldsymbol{c}$ instead of $E, c$ (see Lemma 1.10). The lemma is proved.

Lemma 1.12. In the setup of Lemma 1.11 we have

$$
\phi\left(v^{a-a^{\prime}} T_{w_{0}}\right) t_{x}=\sum_{x^{\prime} \in \boldsymbol{c}} m_{x^{\prime}, x} t_{x^{\prime}}
$$

and

$$
\phi\left(v^{2 a-2 a^{\prime}} T_{w_{0}}^{2}\right) t_{x}=t_{x}
$$

for any $x \in \boldsymbol{c}$, where $m_{x^{\prime}, x} \in \mathbb{Z}$.

Now (b) and the fact that (a) holds with $m_{x^{\prime}, x} \in \mathbb{C}$ is just a restatement of Lemma 1.11. Since $\phi\left(v^{a-a^{\prime}} T_{w_{0}}\right) \in J_{\mathcal{A}}$ we have also $m_{x^{\prime}, x} \in \mathcal{A}$. We now use that $\mathcal{A} \cap \mathbb{C}=\mathbb{Z}$ and the lemma follows.

Lemma 1.13. In the setup of Lemma 1.11 we have for any $x \in \boldsymbol{c}$ the equalities

$$
v^{a-a^{\prime}} T_{w_{0}} c_{x}^{\dagger}=\sum_{x^{\prime} \in \boldsymbol{c}} m_{x^{\prime}, x} c_{x^{\prime}}^{\dagger}
$$

and

$$
v^{2 a-2 a^{\prime}} T_{w_{0}}^{2} c_{x}^{\dagger}=c_{x}^{\dagger}
$$

in $\mathcal{H}^{c}$, where $m_{x^{\prime}, x} \in \mathbb{Z}$ are the same as in Lemma 1.12. Moreover, if $m_{x^{\prime}, x} \neq 0$ then $x^{\prime} \sim_{\mathcal{L}} x$.

The first sentence follows from Lemma 1.12 using [Lusztig 2003, 18.10(a)]. Clearly, if $m_{x^{\prime}, x} \neq 0$ then $x^{\prime} \leq_{\mathcal{L}} x$, which together with $x^{\prime} \sim_{\mathcal{L} \mathcal{R}} x$ implies $x^{\prime} \sim_{\mathcal{L}} x$.

\section{The main results}

2.1. In this section we fix a two-sided cell $c$ of $W ; a, a^{\prime}$ are as in Lemma 1.11. We define an $\mathcal{A}$-linear map $\theta: \mathcal{H}^{\leq \boldsymbol{c}} \rightarrow \mathcal{A}$ by $\theta\left(c_{x}^{\dagger}\right)=1$ if $x \in \mathcal{D} \cap \boldsymbol{c}, \theta\left(c_{x}^{\dagger}\right)=0$ if $x \leq_{\mathcal{L R}} x^{\prime}$ for some $x^{\prime} \in \boldsymbol{c}$ and $x \notin \mathcal{D} \cap \boldsymbol{c}$. Note that $\theta$ is zero on $\mathcal{H}^{<\boldsymbol{c}}$ hence it can be viewed as an $\mathcal{A}$-linear map $\mathcal{H}^{c} \rightarrow \mathcal{A}$.

Lemma 2.2. Let $x, x^{\prime} \in \boldsymbol{c}$. We have

$$
\theta\left(c_{x^{-1}}^{\dagger} c_{x^{\prime}}^{\dagger}\right)=n_{d_{x}} \delta_{x, x^{\prime}} v^{a}+\text { strictly lower powers of } v \text {. }
$$


The left-hand side of (a) is

$$
\begin{aligned}
\sum_{d \in \mathcal{D} \cap c} h_{x^{-1}, x^{\prime}, d} & =\sum_{d \in \mathcal{D} \cap c} \gamma_{x^{-1}, x^{\prime}, d} v^{a}+\text { strictly lower powers of } v \\
& =n_{d_{x}} \delta_{x, x^{\prime}} v^{a}+\text { strictly lower powers of } v .
\end{aligned}
$$

The lemma is proved.

We now state one of the main results of this paper.

Theorem 2.3. There exists a unique permutation $u \mapsto u^{*}$ of $c$ (with square 1) such that for any $u \in c$ we have

$$
v^{a-a^{\prime}} T_{w_{0}} c_{u}^{\dagger}=\epsilon_{u} c_{u^{*}}^{\dagger} \bmod \mathcal{H}^{<c}
$$

where $\epsilon_{u}= \pm 1$. For any $u \in \boldsymbol{c}$ we have $\epsilon_{u^{-1}}=\epsilon_{u}=\epsilon_{\sigma(u)}=\epsilon_{u^{*}}$ and $\sigma\left(u^{*}\right)=$ $(\sigma(u))^{*}=\left(\left(u^{-1}\right)^{*}\right)^{-1}$.

Let $u \in \boldsymbol{c}$. We set $Z=\theta\left(\left(v^{a-a^{\prime}} T_{w_{0}} c_{u}^{\dagger}\right)^{b} v^{a-a^{\prime}} T_{w_{0}} c_{u}^{\dagger}\right)$. We compute $Z$ in two ways, using Lemma 2.2 and Lemma 1.13. We have

$$
\begin{aligned}
Z & =\theta\left(c_{u^{-1}}^{\dagger} v^{2 a-2 a^{\prime}} T_{w_{0}}^{2} c_{u}^{\dagger}\right)=\theta\left(c_{u^{-1}}^{\dagger} c_{u}^{\dagger}\right)=n_{d_{u}} v^{a}+\text { strictly lower powers of } v \\
Z & =\theta\left(\left(\sum_{y \in c} m_{y, u} c_{y}^{\dagger}\right)^{b} \sum_{y^{\prime} \in c} m_{y^{\prime}, u} c_{y^{\prime}}^{\dagger}\right)=\sum_{y, y^{\prime} \in c} m_{y, u} m_{y^{\prime}, u} \theta\left(c_{y^{-1}}^{\dagger} c_{y^{\prime}}^{\dagger}\right) \\
& =\sum_{y, y^{\prime} \in c} m_{y, u} m_{y^{\prime}, u} n_{d_{y}} \delta_{y, y^{\prime}} v^{a}+\text { strictly lower powers of } v \\
& =\sum_{y \in c} n_{d_{y}} m_{y, u}^{2} v^{a}+\text { strictly lower powers of } v \\
& =\sum_{y \in c} n_{d_{u}} m_{y, u}^{2} v^{a}+\text { strictly lower powers of } v
\end{aligned}
$$

where $m_{y, u} \in \mathbb{Z}$ is zero unless $y \sim_{\mathcal{L}} u$ (see Lemma 1.13), in which case we have $d_{y}=d_{u}$. We deduce that $\sum_{y \in c} m_{y, u}^{2}=1$, so that we have $m_{y, u}= \pm 1$ for a unique $y \in \boldsymbol{c}$ (denoted by $u^{*}$ ) and $m_{y, u}=0$ for all $y \in \boldsymbol{c}-\left\{u^{*}\right\}$. Then 2.3(a) holds. Using 2.3(a) and Lemma 1.13(b) we see that $u \mapsto u^{*}$ has square 1 and that $\epsilon_{u} \epsilon_{u^{*}}=1$.

The automorphism $\sigma: \mathcal{H} \rightarrow \mathcal{H}$ (see Section 1.1) satisfies the equality $\sigma\left(c_{u}^{\dagger}\right)=c_{\sigma(u)}^{\dagger}$ for any $u \in W$; note also that $w \in \boldsymbol{c} \leftrightarrow \sigma(w) \in \boldsymbol{c}$ (see Lemma 1.2). Applying $\sigma$ to 2.3(a) we obtain

$$
v^{a-a^{\prime}} T_{w_{0}} c_{\sigma(u)}^{\dagger}=\epsilon_{u} c_{\sigma\left(u^{*}\right)}^{\dagger}
$$

in $\mathcal{H}^{c}$. By 2.3(a) we have also $v^{a-a^{\prime}} T_{w_{0}} c_{\sigma(u)}^{\dagger}=\epsilon_{\sigma(u)} c_{(\sigma(u))^{*}}^{\dagger}$ in $\mathcal{H}^{c}$. It follows that $\epsilon_{u} c_{\sigma\left(u^{*}\right)}^{\dagger}=\epsilon_{\sigma(u)} c_{(\sigma(u))^{*}}^{\dagger}$ hence $\epsilon_{u}=\epsilon_{\sigma(u)}$ and $\sigma\left(u^{*}\right)=(\sigma(u))^{*}$.

Applying $h \mapsto h^{\text {b }}$ to 2.3(a) we obtain

$$
v^{a-a^{\prime}} c_{u^{-1}}^{\dagger} T_{w_{0}}=\epsilon_{u} c_{\left(u^{*}\right)^{-1}}^{\dagger}
$$


in $\mathcal{H}^{c}$. By 2.3(a) we have also

$$
v^{a-a^{\prime}} c_{u^{-1}}^{\dagger} T_{w_{0}}=v^{a-a^{\prime}} T_{w_{0}} c_{\sigma\left(u^{-1}\right)}^{\dagger}=\epsilon_{\sigma\left(u^{-1}\right)} c_{\left(\sigma\left(u^{-1}\right)\right)^{*}}^{\dagger}
$$

in $\mathcal{H}^{c}$. It follows that $\epsilon_{u} c_{\left(u^{*}\right)^{-1}}^{\dagger}=\epsilon_{\sigma\left(u^{-1}\right)} c_{\left(\sigma\left(u^{-1}\right)\right)^{*}}^{\dagger}$ hence $\epsilon_{u}=\epsilon_{\sigma\left(u^{-1}\right)}$ and $\left(u^{*}\right)^{-1}=$ $\left(\sigma\left(u^{-1}\right)\right)^{*}$. Since $\epsilon_{\sigma\left(u^{-1}\right)}=\epsilon_{u^{-1}}$, we see that $\epsilon_{u}=\epsilon_{u^{-1}}$. Replacing $u$ by $u^{-1}$ in $\left(u^{*}\right)^{-1}=\left(\sigma\left(u^{-1}\right)\right)^{*}$ we obtain $\left(\left(u^{-1}\right)^{*}\right)^{-1}=(\sigma(u))^{*}$ as required. The theorem is proved.

2.4. For $u \in c$ we have

$$
\begin{gathered}
u \sim_{\mathcal{L}} u^{*}, \\
\sigma(u) \sim_{\mathcal{R}} u^{*} .
\end{gathered}
$$

Indeed, (a) follows from Lemma 1.13. To prove (b) it is enough to show that $\sigma(u)^{-1} \sim_{\mathcal{L}}\left(u^{*}\right)^{-1}$. Using (a) for $\sigma(u)^{-1}$ instead of $u$ we see that it is enough to show that $\left(\sigma\left(u^{-1}\right)\right)^{*}=\left(u^{*}\right)^{-1}$; this follows from Theorem 2.3.

If we assume that

(c) any left cell in $\boldsymbol{c}$ intersects any right cell in $\mathrm{c}$ in exactly one element then by (a), (b), for any $u \in c$,

(d) $u^{*}$ is the unique element of $\boldsymbol{c}$ in the intersection of the left cell of $u$ with right cell of $\sigma(u)$.

Note that condition (c) is satisfied for any $c$ if $W$ is of type $A_{n}$ or if $W$ is of type $B_{n}(n \geq 2)$ with $L(s)=2$ for all but one $s \in S$ and $L(s)=1$ or 3 for the remaining $s \in S$. (In this last case we are in the quasisplit case and we have $\sigma=1$ hence $u^{*}=u$ for all $u$.)

Theorem 2.5. For any $x \in W$ we set $\vartheta(x)=\gamma_{w_{0} d_{w_{0} x^{-1}}, x,\left(x^{*}\right)^{-1}}$.

(a) If $d \in \mathcal{D}$ and $x, y \in \boldsymbol{c}$ satisfy $\gamma_{w_{0} d, x, y} \neq 0$ then $y=\left(x^{*}\right)^{-1}$.

(b) If $x \in \boldsymbol{c}$ then there is a unique $d \in \mathcal{D} \cap w_{0} \boldsymbol{c}$ such that $\gamma_{w_{0} d, x,\left(x^{*}\right)^{-1}} \neq 0$, namely $d=d_{w_{0} x^{-1}}$. Moreover we have $\vartheta(x)= \pm 1$.

(c) For $u \in c$ we have $\epsilon_{u}=(-1)^{l\left(w_{0} d\right)} n_{d} \vartheta(u)$ where $d=d_{w_{0} u^{-1}}$.

Applying $h \mapsto h^{\dagger}$ to 2.3(a) we obtain for any $u \in \boldsymbol{c}$ :

$$
v^{a-a^{\prime}}(-1)^{l\left(w_{0}\right)} \overline{T_{w_{0}}} c_{u}=\sum_{z \in \boldsymbol{c}} \delta_{z, u^{*}} \epsilon_{u} c_{z} \quad \bmod \sum_{z^{\prime} \in W-\boldsymbol{c}} \mathcal{A} c_{z^{\prime}} .
$$

We have $T_{w_{0}}=\sum_{y \in W}(-1)^{l\left(w_{0} y\right)} p_{1, w_{0} y} c_{y}$ hence $\overline{T_{w_{0}}}=\sum_{y \in W}(-1)^{l\left(w_{0} y\right)} \overline{p_{1, w_{0} y}} c_{y}$. Introducing this in (d) we obtain

$$
v^{a-a^{\prime}} \sum_{y \in W}(-1)^{l(y)} \overline{p_{1, w_{0} y}} c_{y} c_{u}=\sum_{z \in \boldsymbol{c}} \delta_{z, u^{*}} \epsilon_{u} c_{z} \quad \bmod \sum_{z^{\prime} \in W-\boldsymbol{c}} \mathcal{A} c_{z^{\prime}},
$$


that is,

$$
v^{a-a^{\prime}} \sum_{y, z \in W}(-1)^{l(y)} \overline{p_{1, w_{0} y}} h_{y, u, z} c_{z}=\sum_{z \in c} \delta_{z, u^{*}} \epsilon_{u} c_{z} \quad \bmod \sum_{z^{\prime} \in W-c} \mathcal{A} c_{z^{\prime}} .
$$

Thus, for $z \in c$ we have

$$
v^{a-a^{\prime}} \sum_{y \in W}(-1)^{l(y)} \overline{p_{1, w_{0} y}} h_{y, u, z}=\delta_{z, u^{*}} \epsilon_{u} .
$$

Here we have $h_{y, u, z}=\gamma_{y, u, z^{-1}} v^{-a} \bmod v^{-a+1} \mathbb{Z}[v]$ and we can assume than $z \leq_{\mathcal{R}} y$ so that $w_{0} y \leq_{\mathcal{R}} w_{0} z$ and $\boldsymbol{a}\left(w_{0} y\right) \geq \boldsymbol{a}\left(w_{0} z\right)=a^{\prime}$.

For $w \in W$ we set $s_{w}=n_{w}$ if $w \in \mathcal{D}$ and $s_{w}=0$ if $w \notin \mathcal{D}$. By [Lusztig 2003, 14.1] we have $p_{1, w}=s_{w} v^{-\boldsymbol{a}(w)} \bmod v^{-\boldsymbol{a}(w)-1} \mathbb{Z}\left[v^{-1}\right]$ hence $\overline{p_{1, w}}=s_{w} v^{\boldsymbol{a}(w)}$ $\bmod v^{\boldsymbol{a}(w)+1} \mathbb{Z}[v]$. Hence for $y$ in the sum above we have $\overline{p_{1, w_{0} y}}=s_{w_{0} y} v^{\boldsymbol{a}\left(w_{0} y\right)}$ $\bmod v^{\boldsymbol{a}\left(w_{0} y\right)+1} \mathbb{Z}[v]$. Thus (e) gives

$$
v^{a-a^{\prime}} \sum_{y \in \boldsymbol{c}}(-1)^{l(y)} s_{w_{0} y} \gamma_{y, u, z^{-1}} v^{a\left(w_{0} y\right)-a}-\delta_{z, u^{*}} \epsilon_{u} \in v \mathbb{Z}[v]
$$

and using $\boldsymbol{a}\left(w_{0} y\right)=a^{\prime}$ for $y \in \boldsymbol{c}$ we obtain

$$
\sum_{y \in c}(-1)^{l(y)} s_{w_{0} y} \gamma_{y, u, z^{-1}}=\delta_{z, u^{*}} \epsilon_{u} .
$$

Using the definition of $s_{w_{0} y}$ we obtain

$$
\sum_{d \in \mathcal{D} \cap w_{0} c}(-1)^{l\left(w_{0} d\right)} n_{d} \gamma_{w_{0} d, u, z^{-1}}=\delta_{z, u^{*}} \epsilon_{u}
$$

Next we note that

$$
\text { if } d \in \mathcal{D} \text { and } x, y \in c \text { satisfy } \gamma_{w_{0} d, x, y} \neq 0 \text { then } d=d_{w_{0} x^{-1}} \text {. }
$$

Indeed from [Lusztig 2003, §14, P8] we deduce $w_{0} d \sim_{\mathcal{L}} x^{-1}$. Using [Lusztig 2003, 11.7] we deduce $d \sim_{\mathcal{L}} w_{0} x^{-1}$ so that $d=d_{w_{0}^{-1} x^{-1}}$. This proves $(\mathrm{g})$.

Using (g) we can rewrite (f) as follows.

$$
(-1)^{l\left(w_{0}\right)}(-1)^{l(d)} n_{d} \gamma_{w_{0} d, u, z^{-1}}=\delta_{z, u^{*}} \epsilon_{u}
$$

where $d=d_{w_{0} u^{-1}}$.

We prove (a). Assume that $d \in \mathcal{D}$ and $x, y \in c$ satisfy $\gamma_{w_{0} d, x, y} \neq 0, y \neq\left(x^{*}\right)^{-1}$. Using (g) we have $d=d_{w_{0} x^{-1}}$. Using (h) with $u=x, z=y^{-1}$ we see that $\gamma_{w_{0} d, x, y}=0$, a contradiction. This proves (a).

We prove (b). Using (h) with $u=x, z=x^{*}$ we see that

$$
(-1)^{l\left(w_{0} d\right)} n_{d} \gamma_{w_{0} d, x,\left(x^{*}\right)^{-1}}=\epsilon_{u}
$$


where $d=d_{w_{0} x^{-1}}$. Hence the existence of $d$ in (b) and the equality $\vartheta(x)= \pm 1$ follow; the uniqueness of $d$ follows from (g).

Now (c) follows from (i). This completes the proof of the theorem.

2.6. In the case where $L=l, \vartheta(u)$ (in $2.5(\mathrm{c})$ ) is $\geq 0$ and \pm 1 hence 1 ; moreover, $n_{d}=1,(-1)^{l(d)}=(-1)^{a^{\prime}}$ for any $d \in \mathcal{D} \cap w_{0} c$ (by the definition of $\mathcal{D}$ ). Hence we have $\epsilon_{u}=(-1)^{l\left(w_{0}\right)+a^{\prime}}$ for any $u \in \boldsymbol{c}$, a result of Mathas [1996].

Now Theorem 2.5 also gives a characterization of $u^{*}$ for $u \in \boldsymbol{c}$; it is the unique element $u^{\prime} \in \boldsymbol{c}$ such that $\gamma_{w_{0} d, u, u^{\prime-1}} \neq 0$ for some $d \in \mathcal{D} \cap w_{0} \boldsymbol{c}$.

We will show:

(a) The subsets $X=\left\{d^{*} ; d \in \mathcal{D} \cap \boldsymbol{c}\right\}$ and $X^{\prime}=\left\{w_{0} d^{\prime} ; d^{\prime} \in \mathcal{D} \cap w_{0} c\right\}$ of c coincide.

Let $d \in \mathcal{D} \cap \boldsymbol{c}$. By 2.5(b) we have $\gamma_{w_{0} d^{\prime}, d,\left(d^{*}\right)^{-1}}= \pm 1$ for some $d^{\prime} \in \mathcal{D} \cap w_{0} c$. Hence $\gamma_{\left(d^{*}\right)^{-1}, w_{0} d^{\prime}, d}= \pm 1$. Using [Lusztig 2003, 14.2, P2] we deduce $d^{*}=w_{0} d^{\prime}$. Thus $X \subset X^{\prime}$. Let $Y$ (resp. $Y^{\prime}$ ) be the set of left cells contained in $\boldsymbol{c}$ (resp. $w_{0} \boldsymbol{c}$ ). We have $\sharp(X)=\sharp(Y)$ and $\sharp\left(X^{\prime}\right)=\sharp\left(Y^{\prime}\right)$. By [Lusztig 2003, 11.7(c)] we have $\sharp(Y)=\sharp\left(Y^{\prime}\right)$. It follows that $\sharp(X)=\sharp\left(X^{\prime}\right)$. Since $X \subset X^{\prime}$, we must have $X=X^{\prime}$. This proves (a).

\section{Theorem 2.7. We have}

$$
\phi\left(v^{a-a^{\prime}} T_{w_{0}}\right)=\sum_{d \in \mathcal{D} \cap c} \vartheta(d) \epsilon_{d} t_{d^{*}} \bmod \sum_{u \in W-c} \mathcal{A} t_{u} .
$$

We set $\phi\left(v^{a-a^{\prime}} T_{w_{0}}\right)=\sum_{u \in W} p_{u} t_{u}$ where $p_{u} \in \mathcal{A}$. Combining 1.12a, 1.13a, 2.3(a) we see that for any $x \in c$ we have

$$
\phi\left(v^{a-a^{\prime}} T_{w_{0}}\right) t_{x}=\epsilon_{x} t_{x^{*}}
$$

hence

$$
\epsilon_{x} t_{x^{*}}=\sum_{u \in c} p_{u} t_{u} t_{x}=\sum_{u, y \in c} p_{u} \gamma_{u, x, y^{-1}} t_{y}
$$

It follows that for any $x, y \in \boldsymbol{c}$ we have

$$
\sum_{u \in c} p_{u} \gamma_{u, x, y^{-1}}=\delta_{y, x^{*}} \epsilon_{x} .
$$

Taking $x=w_{0} d$ where $d=d_{w_{0} y} \in \mathcal{D} \cap w_{0} c$ we obtain

$$
\sum_{u \in \boldsymbol{c}} p_{u} \gamma_{w_{0} d_{w_{0} y}, y^{-1}, u}=\delta_{y,\left(w_{0} d_{w_{0} y}\right)^{*}} \epsilon_{w_{0} d_{w_{0} y}}
$$

which, by Theorem 2.5 , can be rewritten as

$$
p_{\left(\left(y^{-1}\right)^{*}\right)^{-1} \vartheta}\left(y^{-1}\right)=\delta_{y,\left(w_{0} d_{w_{0} y}\right)^{*}} \epsilon_{w_{0} d_{w_{0} y}} .
$$


We see that for any $y \in \boldsymbol{c}$ we have

$$
p_{\sigma\left(y^{*}\right)}=\delta_{y,\left(w_{0} d_{w_{0} y}\right)^{*} \vartheta}\left(y^{-1}\right) \epsilon_{w_{0} d_{w_{0}}} .
$$

In particular we have $p_{\sigma\left(y^{*}\right)}=0$ unless $y=\left(w_{0} d_{w_{0} y}\right)^{*}$ in which case

$$
p_{\sigma\left(y^{*}\right)}=p_{\left.(\sigma(y))^{*}\right)}=\vartheta\left(y^{-1}\right) \epsilon_{y} .
$$

(We use that $\epsilon_{y^{*}}=\epsilon_{y}$.) If $y=\left(w_{0} d_{w_{0} y}\right)^{*}$ then $y^{*} \in X^{\prime}$ hence by 2.6(a), $y^{*}=d^{*}$ that is $y=d$ for some $d \in \mathcal{D}$. Conversely, if $y \in \mathcal{D}$ then $w_{0} y^{*} \in \mathcal{D}$ (by 2.6(a)) and $w_{0} y^{*} \sim_{\mathcal{L}} w_{0} y$ (since $\left.y^{*} \sim_{\mathcal{L}} y\right)$ hence $d_{w_{0} y}=w_{0} y^{*}$. We see that $y=\left(w_{0} d_{w_{0} y}\right)^{*}$ if and only if $y \in \mathcal{D}$. We see that

$$
\phi\left(v^{a-a^{\prime}} T_{w_{0}}\right)=\sum_{d \in \mathcal{D} \cap c} \vartheta\left(d^{-1}\right) \epsilon_{d} t_{(\sigma(d))^{*}}+\sum_{u \in W-c} p_{u} t_{u} .
$$

Now $d \mapsto \sigma(d)$ is a permutation of $\mathcal{D} \cap \boldsymbol{c}$ and $\vartheta\left(d^{-1}\right)=\vartheta(d)=\vartheta(\sigma(d)), \epsilon_{\sigma(d)}=\epsilon_{d}$. The theorem follows.

Corollary 2.8. $\quad \phi\left(T_{w_{0}}\right)=\sum_{d \in \mathcal{D}} \vartheta(d) \epsilon_{d} v^{-\boldsymbol{a}(d)+\boldsymbol{a}\left(w_{0} d\right)} t_{d^{*}} \in J_{\mathcal{A}}$

2.9. We set $\mathfrak{T}_{\boldsymbol{c}}=\sum_{d \in \mathcal{D} \cap \boldsymbol{c}} \vartheta(d) \epsilon_{d} t_{d^{*}} \in J^{c}$. We show:

(a) $\mathfrak{T}_{c}^{2}=\sum_{d \in \mathcal{D} \cap c} n_{d} t_{d}$.

(b) $t_{x} \mathfrak{T}_{c}=\mathfrak{T}_{c} t_{\sigma(x)}$ for any $x \in W$.

By Theorem 2.7 we have $\phi\left(v^{a-a^{\prime}} T_{w_{0}}\right)=\mathfrak{T}_{c}+\xi$ where $\xi \in J_{K}^{W-c}:=\sum_{u \in W-c} K t_{u}$. Since $J_{K}^{c}, J_{K}^{W-c}$ are two-sided ideals of $J_{K}$ with intersection zero and $\phi_{K}: \mathcal{H}_{K} \rightarrow J_{K}$ is an algebra homomorphism, it follows that

$$
\phi\left(v^{2 a-2 a^{\prime}} T_{w_{0}}^{2}\right)=\left(\phi\left(v^{a-a^{\prime}} T_{w_{0}}\right)\right)^{2}=\left(\mathfrak{T}_{c}+\xi\right)^{2}=\mathfrak{T}_{c}^{2}+\xi^{\prime}
$$

where $\xi^{\prime} \in J_{K}^{W-c}$. Hence, for any $x \in \boldsymbol{c}$ we have $\phi\left(v^{2 a-2 a^{\prime}} T_{w_{0}}^{2}\right) t_{x}=\mathfrak{T}_{c}^{2} t_{x}$ so that (using 1.12b): $t_{x}=\mathfrak{T}_{c}^{2} t_{x}$. We see that $\mathfrak{T}_{c}^{2}$ is the unit element of the ring $J_{K}^{c}$. Thus (a) holds.

We prove (b). For any $y \in W$ we have $T_{y} T_{w_{0}}=T_{w_{0}} T_{\sigma(y)}$ hence, applying $\phi_{K}$,

$$
\phi\left(T_{y}\right) \phi\left(v^{a-a^{\prime}} T_{w_{0}}\right)=\phi\left(v^{a-a^{\prime}} T_{w_{0}}\right) \phi\left(T_{\sigma(y)}\right),
$$

that is, $\phi\left(T_{y}\right)\left(\mathfrak{T}_{c}+\xi\right)=\left(\mathfrak{T}_{c}+\xi\right) \phi\left(T_{\sigma(y)}\right)$. Thus, $\phi\left(T_{y}\right) \mathfrak{T}_{c}=\mathfrak{T}_{c} \phi\left(T_{\sigma(y)}\right)+\xi_{1}$ where $\xi_{1} \in J_{K}^{W-c}$. Since $\phi_{K}$ is an isomorphism, it follows that for any $x \in W$ we have $t_{x} \mathfrak{T}_{\boldsymbol{c}}=\mathfrak{T}_{c} t_{\sigma(x)} \bmod J_{K}^{W-c}$. Thus (b) holds. 
2.10. In this subsection we assume that $L=l$. In this case Corollary 2.8 becomes

$$
\phi\left(T_{w_{0}}\right)=\sum_{d \in \mathcal{D}}(-1)^{l\left(w_{0}\right)+\boldsymbol{a}\left(w_{0} d\right)} v^{-\boldsymbol{a}(d)+\boldsymbol{a}\left(w_{0} d\right)} t_{d^{*}} \in J_{\mathcal{A}} .
$$

(We use that $\vartheta(d)=1$.)

For any left cell $\Gamma$ contained in $c$ let $n_{\Gamma}$ be the number of fixed points of the permutation $u \mapsto u^{*}$ of $\Gamma$. Now $\Gamma$ carries a representation $[\Gamma]$ of $W$ and from Theorem 2.3 we see that $\operatorname{tr}\left(w_{0},[\Gamma]\right)= \pm n_{\Gamma}$. Thus $n_{\Gamma}$ is the absolute value of the integer $\operatorname{tr}\left(w_{0},[\Gamma]\right)$. From this the number $n_{\Gamma}$ can be computed for any $\Gamma$. In this way we see for example that if $W$ is of type $E_{7}$ or $E_{8}$ and $c$ is not an exceptional two-sided cell, then $n_{\Gamma}>0$.

\section{Acknowledgements}

I thank Matthew Douglass for bringing the paper [Mathas 1996] to my attention. I thank the referee for helpful comments.

\section{References}

[Bezrukavnikov et al. 2012] R. Bezrukavnikov, M. Finkelberg, and V. Ostrik, "Character D-modules via Drinfeld center of Harish-Chandra bimodules", Invent. Math. 188 (2012), 589-620. MR 2917178 Zbl 1267.20058

[Elias and Williamson 2014] B. Elias and G. Williamson, "The Hodge theory of Soergel bimodules", Ann. of Math. (2) 180:3 (2014), 1089-1136. MR 3245013 Zbl 06380813

[Kazhdan and Lusztig 1979] D. Kazhdan and G. Lusztig, "Representations of Coxeter groups and Hecke algebras”, Invent. Math. 53:2 (1979), 165-184. MR 81j:20066 Zbl 0499.20035

[Lusztig 1984] G. Lusztig, Characters of reductive groups over a finite field, Annals of Mathematics Studies 107, Princeton University Press, Princeton, NJ, 1984. MR 86j:20038 Zbl 0556.20033

[Lusztig 1990] G. Lusztig, "Canonical bases arising from quantized enveloping algebras. II", Progr. Theoret. Phys. Suppl. 102 (1990), 175-201. MR 93g:17019 Zbl 0776.17012

[Lusztig 2003] G. Lusztig, Hecke algebras with unequal parameters, CRM Monograph Series 18, American Mathematical Society, Providence, RI, 2003. MR 2004k:20011 Zbl 1051.20003

[Lusztig 2005] G. Lusztig, "Character sheaves on disconnected groups. VII", Represent. Theory 9 (2005), 209-266. MR 2006e:20089 Zbl 1078.20047

[Mathas 1996] A. Mathas, "On the left cell representations of Iwahori-Hecke algebras of finite Coxeter groups", J. London Math. Soc. (2) 54:3 (1996), 475-488. MR 97h:20008 Zbl 0865.20027

Received March 3, 2015.

GEORGE LuSZTiG

DEPARTMENT OF MATHEMATICS

MassachusetTs Institute of TeChNOLOGY

CAMBRIDGE, MA 02139-4307

UNITED STATES

gyuri@math.mit.edu 


\title{
PACIFIC JOURNAL OF MATHEMATICS
}

\author{
msp.org/pjm
}

Founded in 1951 by E. F. Beckenbach (1906-1982) and F. Wolf (1904-1989)

\section{EDITORS}

Don Blasius (Managing Editor)

Department of Mathematics

University of California

Los Angeles, CA 90095-1555

blasius@math.ucla.edu

\author{
Paul Balmer \\ Department of Mathematics \\ University of California \\ Los Angeles, CA 90095-1555 \\ balmer@math.ucla.edu \\ Robert Finn \\ Department of Mathematics \\ Stanford University \\ Stanford, CA 94305-2125 \\ finn@math.stanford.edu \\ Sorin Popa \\ Department of Mathematics \\ University of California \\ Los Angeles, CA 90095-1555 \\ popa@math.ucla.edu
}

\author{
Vyjayanthi Chari \\ Department of Mathematics \\ University of California \\ Riverside, CA 92521-0135 \\ chari@math.ucr.edu \\ Kefeng Liu \\ Department of Mathematics \\ University of California \\ Los Angeles, CA 90095-1555 \\ liu@math.ucla.edu \\ Jie Qing \\ Department of Mathematics \\ University of California \\ Santa Cruz, CA 95064 \\ qing@ cats.ucsc.edu
}

\section{PRODUCTION}

Silvio Levy, Scientific Editor, production@msp.org

\section{SUPPORTING INSTITUTIONS}

ACADEMIA SINICA, TAIPEI

CALIFORNIA INST. OF TECHNOLOGY

INST. DE MATEMÁTICA PURA E APLICADA

KEIO UNIVERSITY

MATH. SCIENCES RESEARCH INSTITUTE

NEW MEXICO STATE UNIV.

OREGON STATE UNIV.

\author{
STANFORD UNIVERSITY \\ UNIV. OF BRITISH COLUMBIA \\ UNIV. OF CALIFORNIA, BERKELEY \\ UNIV. OF CALIFORNIA, DAVIS \\ UNIV. OF CALIFORNIA, LOS ANGELES \\ UNIV. OF CALIFORNIA, RIVERSIDE \\ UNIV. OF CALIFORNIA, SAN DIEGO \\ UNIV. OF CALIF., SANTA BARBARA
}

\author{
Daryl Cooper \\ Department of Mathematics \\ University of California \\ Santa Barbara, CA 93106-3080 \\ cooper@math.ucsb.edu \\ Jiang-Hua Lu \\ Department of Mathematics \\ The University of Hong Kong \\ Pokfulam Rd., Hong Kong \\ jhlu@maths.hku.hk \\ Paul Yang \\ Department of Mathematics \\ Princeton University \\ Princeton NJ 08544-1000 \\ yang@math.princeton.edu
}

These supporting institutions contribute to the cost of publication of this Journal, but they are not owners or publishers and have no responsibility for its contents or policies.

See inside back cover or msp.org/pjm for submission instructions.

The subscription price for 2015 is US \$420/year for the electronic version, and \$570/year for print and electronic.

Subscriptions, requests for back issues and changes of subscribers address should be sent to Pacific Journal of Mathematics, P.O. Box 4163, Berkeley, CA 94704-0163, U.S.A. The Pacific Journal of Mathematics is indexed by Mathematical Reviews, Zentralblatt MATH, PASCAL CNRS Index, Referativnyi Zhurnal, Current Mathematical Publications and Web of Knowledge (Science Citation Index).

The Pacific Journal of Mathematics (ISSN 0030-8730) at the University of California, c/o Department of Mathematics, 798 Evans Hall \#3840, Berkeley, CA 94720-3840, is published twelve times a year. Periodical rate postage paid at Berkeley, CA 94704, and additional mailing offices. POSTMASTER: send address changes to Pacific Journal of Mathematics, P.O. Box 4163, Berkeley, CA 94704-0163.

PJM peer review and production are managed by EditFLOW ${ }^{\circledR}$ from Mathematical Sciences Publishers.

\section{PUBLISHED BY}

\section{mathematical sciences publishers \\ nonprofit scientific publishing}

http://msp.org/

(C) 2015 Mathematical Sciences Publishers 


\title{
PACIFIC JOURNAL OF MATHEMATICS
}

\author{
Volume 279 No. 1-2 December 2015
}

In memoriam: Robert Steinberg

Robert Steinberg (1922-2014): In memoriam V. S. VARADARAJAN

Cellularity of certain quantum endomorphism algebras

HENNING H. ANDERSEN, GUSTAV I. LEHRER and RUIBIN ZHANG

Lower bounds for essential dimensions in characteristic 2 via orthogonal representations ANTONIO BABIC and VLADIMIR CHERNOUSOV

Cocharacter-closure and spherical buildings

Michael Bate, Sebastian Herpel, Benjamin Martin and Gerhard RöHrLe

Embedding functor for classical groups and Brauer-Manin obstruction

Eva Bayer-Fluckiger, Ting-Yu LeE and Raman Parimala

On maximal tori of algebraic groups of type $G_{2}$

Constantin Beli, PhilipPe Gille and Ting-Yu LeE

On extensions of algebraic groups with finite quotient

MICHEL BRION

Essential dimension and error-correcting codes

SHANE CERNELE and ZiNOVy REICHSTEIN

Notes on the structure constants of Hecke algebras of induced representations of finite Chevalley groups

Charles W. CuRTis

Complements on disconnected reductive groups

FRANÇOIS DIGNE and JEAN MICHEL

Extending Hecke endomorphism algebras

Jie Du, Brian J. Parshall and LeOnard L. SCOTT

Products of partial normal subgroups

ELLEN HENKE

Lusztig induction and $\ell$-blocks of finite reductive groups

RADHA KESSAR and GUNTER MALLE

Free resolutions of some Schubert singularities

Manoj Kummini, Venkatramani Lakshmibai, Pramathanath Sastry and C. S. Seshadri

Free resolutions of some Schubert singularities in the Lagrangian Grassmannian

VenKatramani LAKSHMibai and ReUVEN HODGES

Distinguished unipotent elements and multiplicity-free subgroups of simple algebraic groups

Martin W. Liebeck, Gary M. Seitz and Donna M. Testerman

Action of longest element on a Hecke algebra cell module

GEORGE LUSZTIG

Generic stabilisers for actions of reductive groups

BENJAMIN MARTIN

On the equations defining affine algebraic groups

VLADIMIR L. POPOV

Smooth representations and Hecke modules in characteristic $p$

PETER SCHNEIDER

On CRDAHA and finite general linear and unitary groups

BHAMA SRINIVASAN

Weil representations of finite general linear groups and finite special linear groups PHAM HUU TIEP

The pro- $p$ Iwahori Hecke algebra of a reductive $p$-adic group, $\mathrm{V}$ (parabolic induction) MARIE-FRANCE VIGNÉRAS

Acknowledgement 